\title{
Vortex nucleation in rotating BEC: the role of the boundary condition for the order parameter
}

\author{
W. V. Pogosov and K. Machida \\ Department of Physics, Okayama University, Okayama 700-8530, Japan
}

(Dated: 2nd November 2018)

\begin{abstract}
We study the process of vortex nucleation in rotating two-dimensional BEC confined in a harmonic trap. We show that, within the Gross-Pitaevskii theory with the boundary condition of vanishing of the order parameter at infinity, topological defects nucleation occurs via the creation of vortexantivortex pairs far from the cloud center, where the modulus of the order parameter is small. Then, vortices move towards the center of the cloud and antivortices move in the opposite direction but never disappear. We also discuss the role of surface modes in this process.

PACS numbers: 03.75.Fi, 05.30.Jp, 32.80.-t
\end{abstract}

\section{INTRODUCTION}

Quantized vortices in Bose-Einstein condensates (BEC) of alkali atom gases attract a considerable current interest. One of the most interesting problems of vortex physics is the nucleation of topological defects. A lot of experimental [1, 2, 3, 4] and theoretical [5, 6, 7, 8, 9, 10, 11, 12, 13, 14, 15, 16, 17, 18, 19, 20, 21] works deal with this subject. Phases with different number of vortices are usually separated by large energetic barriers. Therefore, the transition to the vortex state occurs at higher rotation frequency than the thermodynamical one determined by the balance of energies of vortex-free and one-vortex states. The same is valid for the well-studied case of superconductors [23, 24]. However, BEC clouds have no well-defined surface and, therefore, the process of vortex formation must be different from that in superconductors, where vortex lines penetrate from the sample surface. Within the Gross-Pitaevskii theory, the boundary condition for the order parameter in the harmonically trapped BEC reads

$$
\psi(r \rightarrow \infty)=0 .
$$

In the li1 of strongly interacting gas, a Thomas-Fermi approximation is often used. In this approach, the ThomasFermi boundary, where $\psi$ vanishes identically, has to be assumed, and vortices are usually treated as point-like objects. Under these assumptions, one can calculate the critical rotation frequency, which suppresses the surface barrier preventing vortex penetration through the Thomas-Fermi boundary [7, 11, 12. Another approach to the problem 5, 9, 10, 13 is based on the analysis of stability of the axially-symmetric vortex-free state with respect to the perturbations of the order parameter $\psi$, and it was shown that the nonvortex state becomes unstable with respect to surface modes, which leads to the well-known Landau instability. It is believed that this instability results in the nucleation of vortices. The third approach to the problem of vortex formation is by numerical solutions of the time-dependent Gross-Pitaevskii (GP) equation [6, 8, 14, 15, 16], supplemented by the boundary condition of varnishing of $\psi$ at some boundary.

The aim of the present paper is to study a vortex nucleation process taking into account consistently boundary condition (1) for the order parameter. We consider the case, when the system is spined up quasistatically and adiabatically. No boundary, which can serve as a gate for vortex penetration is assumed. We use a semi-analytical model [25], which is applicable for the li1 of diluted gas. We show that nucleation of vortices in the model with boundary condition (1) occurs via the creation of vortex-antivortex pairs far from the cloud center, in regions, where $|\psi|$ is small. Vortices then move toward the cloud center to decrease the energy of the system, whereas antivortices go to the opposite direction. This mechanism for the vortex nucleation is natural for the case of a system, which has no boundary and where the total topological charge has to be conserved. Also studied is the role of surface modes in the process of vortex nucleation. We show that the critical velocity for vortex formation is slightly higher than the Landau critical velocity, and this is in a agreement with Anglin's results [1, 12] obtained by the very different method.

\section{MODEL}

We consider the case of quasi two-dimensional condensate. The dimensionless GP energy functional is given by:

$$
F=\int r d r \int d \varphi\left(\frac{1}{2}|\nabla \psi|^{2}+\frac{r^{2}}{2}|\psi|^{2}+\frac{2 \pi p}{N}|\psi|^{4}+i \omega \psi^{*} \frac{\partial \psi}{\partial \phi}\right)
$$


where the integration is performed over the area of the system, $(r, \varphi)$ are polar coordinates, $N$ is the number of atoms, $\omega$ is the rotation frequency, $p=a n_{z}$ is the gas parameter, $a$ and $n_{z}$ are scattering length and concentration of particles along the cloud axis, respectively. Distances and rotation frequencies are measured in units of the oscillator length and the trapping frequency, respectively. The normalization condition for the order parameter reads $\int r d r \int d \varphi|\psi|^{2}=N$.

In general case, $\psi$ can be represented as a Fourier expansion

$$
\psi(r, \varphi)=\sum_{l} f_{l}(r) \exp (-i l \varphi) .
$$

In the li1 of noninteracting gas $(p=0)$, it can be easily shown by substitution of Eq. (3) to the GP equation that each function $f_{l}(r)$ coincides with the eigen function for the harmonic oscillator corresponding to the angular momentum $l$. These functions have the Gaussian profile $\sim r^{l} \exp \left(-\frac{r^{2}}{2}\right)$. Therefore, one can assume that this Gaussian approximation remains accurate in the case of weakly interacting dilute gas. The accuracy can be improved if we introduce a variational parameter $R_{l}$ characterizing the spatial extend of $f_{l}(r)$. Finally, our ansatz for $f_{l}(r)$ has a form:

$$
f_{l}(r)=C_{l}\left(\frac{r}{R_{l}}\right)^{l} \exp \left(-\frac{r^{2}}{2 R_{l}^{2}}-i \phi_{l}\right),
$$

where $C_{l}, R_{l}$, and $\phi_{l}$ can be found from the condition of minimum of the energy (2), $C_{l}$ is a real number. This approach was used for the first time in Ref. [25], see also Ref. [26, 27]. In the present paper, we restrict ourselves to the interval $0<p<10$, since, according to our estimates, the Gaussian approximation becomes inaccurate at higher $p$. To verify this, we compared known numerical results for the thermodynamical critical rotation frequency with those obtained by our method.

Now we substitute Eqs. (3) and (4) to Eq. (2) and after the integration we obtain:

$$
\begin{aligned}
F= & \sum_{l} \alpha_{l} C_{l}^{2}+\sum_{l} I_{l l l l} C_{l}^{4}+4 \sum_{l>k} I_{l l k k} C_{l}^{2} C_{k}^{2} \\
& +4 \sum_{l>k>m} I_{l k k m} C_{l} C_{k}^{2} C_{m} \delta_{l+m, 2 k} \cos \left(\phi_{l}+\phi_{m}-2 \phi_{k}\right) \\
& +8 \sum_{l>k>m>n} I_{l k m n} C_{l} C_{k} C_{m} C_{n} \delta_{l+k, m+n} \cos \left(\phi_{l}+\phi_{k}-\phi_{m}-\phi_{n}\right),
\end{aligned}
$$

where

$$
\begin{aligned}
& \alpha_{l}=\frac{\pi}{2} \Gamma(l+2)\left(1+R_{l}^{4}\right)+\pi R_{l}^{2} \Gamma(l+1) \omega l \\
I_{l k m n}= & \frac{2 \pi^{2} p}{N} \Gamma\left(\frac{l+m+n+k}{2}+1\right) R_{l k m n}^{2} \\
& \times\left(\frac{R_{l k m n}}{R_{l}}\right)^{l}\left(\frac{R_{l k m n}}{R_{k}}\right)^{k} \times\left(\frac{R_{l k m n}}{R_{m}}\right)^{m}\left(\frac{R_{l k m n}}{R_{n}}\right)^{n} \\
& R_{l k m n}=\sqrt{2}\left(R_{l}^{-2}+R_{k}^{-2}+R_{m}^{-2}+R_{n}^{-2}\right)^{-\frac{1}{2}}
\end{aligned}
$$

$\Gamma(l)$ is a gamma function. Normalization condition is now given by

$$
\pi \sum_{l} C_{l}^{2} R_{l}^{2} \Gamma(l+1)=N
$$

Values of parameters $R_{l}, C_{l}$ and $\phi_{l}$ can be found from the minimum of the energy (5) taking into account Eq. (9). For instance, for the axially-symmetric vortex-free state, $C_{0}=\sqrt{N / \pi R_{0}^{2}}, R_{0}=(1+2 p)^{1 / 4}$, and $C_{l}=0$ at $l>1$.

In the vicinity of the local minimum, the deviation of the energy from the minimum can be represented as

$$
\Delta F=\sum_{l k} A_{l k} \Delta C_{l} \Delta C_{k}
$$

where $\Delta C_{l}$ is a deviation of $C_{l}$ from its equilibrium value. In Eq. (10) we kept the terms up to the second order of $\Delta C_{l}$. The stability criterion of the solution is a positiveness of all main minors of the matrix $\left\|A_{l k}\right\|$. 


\section{STABILITY OF NONVORTEX STATE}

First, we study the stability of the vortex-free state and after this we will go beyond this linear approach. We consider the situation, when the system is spined up quasistatically. For the axially-symmetric nonvortex state, the matrix $\left\|A_{l k}\right\|$ is diagonal. In this case, it is easy to find $\omega$, at which vortex-free phase becomes unstable with respect to the surface mode with the angular momentum $l$ :

$$
\begin{aligned}
\omega_{\text {inst }}(l, p)= & \frac{2}{l \Gamma(l+1)}\left[\frac{(l-1) \Gamma(l+1)}{\sqrt{1+2 p}}+\right. \\
& \frac{1}{4}\left[\frac{1+R_{l}^{4}}{R_{l}^{2}}\right] \Gamma(l+2)+\frac{4(1+2 p)^{l / 2} \Gamma(l+1)}{\left(\sqrt{1+2 p}+R_{l}^{2}\right)^{l+1}} .
\end{aligned}
$$

Value of $R_{l}$ can be found from the condition of minimum of $\omega_{\text {inst }}(l, p)$ with respect to $R_{l}$. In Fig. 1 (a) we plot the dependences of $\omega_{\text {inst }}(l, p)$ on $p$ for different $l$. The resulting function $\omega_{\text {inst }}(p)$ is determined by the minimal value of $\omega_{\text {inst }}(l, p)$ with respect to the quantized $l$. This function is shown in Fig. 1 (b) and represents a well-known Landau criterion for the angular-momentum transfer to the superfluid system. From Fig. 1 (b) one can see that value of $l_{\text {inst }}$, which leads to the instability, increases with increase of gas parameter $p$. Vortex-free phase is always stable with respect to the generation of harmonic $l=1$. At low $p$ values, the instability with respect to the harmonic $l=3$ occurs at lower $\omega$ than that for $l=2$. In Fig. 1 (b), we also presented the thermodynamical critical rotation frequency as a function of $p$, which we calculated by the comparison of energies of vortex-free and single-vortex states. It is much lower than $\omega_{\text {inst }}$, as can be expected.

\section{NUCLEATION OF VORTICES}

Now we go beyond the linear analysis. It follows from the results of the previous Section that, in the instability point, an admixture of a harmonic with $l=l_{\text {inst }}$ appears in the order parameter expansion (3). In fact, the order parameter must contain also all other harmonics with $l$ 's divisible by $l_{\text {inst }}$, which are induced by the component with $l=l_{\text {inst }}$, as can be easily seen from the GP equation. However, the main contribution to the energy is given by just two first terms with $l=0$ and $l=l_{\text {inst }}$. Therefore, we will take into account only these two harmonics. The component with $l=l_{\text {inst }}$ gives $l_{\text {inst }}$-fold modulation of the modulus of the order parameter in the azimuthal direction and it is responsible for the creation of vortices. We calculate the energy and $\psi$ in the two-harmonic approximation at values of $\omega$ slightly exceeding $\omega_{\text {inst }}$ and study their evolution with changing of $\omega$. At each $\omega$ we also check the stability

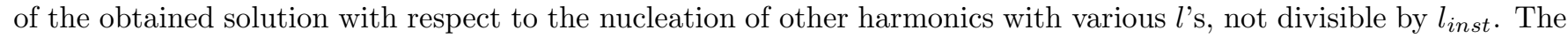
two-harmonic approximation was used widely for the analysis of vortex states in mesoscopic superconducting discs within the Ginzburg-Landau theory, see e.g. Refs. [28, 29, 30, 31]. Note that we also tried to take into account several additional components of $\psi$ with $l$ 's divisible by $l_{\text {inst }}\left(2 l_{\text {inst }}, 3 l_{\text {inst }}, 4 l_{\text {inst }}\right)$, but this does not change our results significantly thus verifying good accuracy of the two-harmonic approximation in our case.

First, we discuss the simplest case of $l_{\text {inst }}=3$. In the instability point, an admixture of harmonic with $l=3$ appears. Surface mode leads to the three-fold modulation of $|\psi|$ in the azimuthal direction. In Fig. 2 we show the evolution of $|\psi|$ far from the system center along one of three directions, where $|\psi|$ is minimal, at $p=1$. In the beginning, $|\psi|$ in this direction is a monotonic function of $r$. With further increasing of $\omega$, a local minimum appears at the dependence of $\psi(r)$, see Fig. 2 (a). This minimum becomes deeper and deeper and then $|\psi|$ vanishes at some point, as shown on Fig. 2 (b). If we proceed with spinning up the system, the minimum splits to the vortex-antivortex pair, see Fig. 2 (c). If we go around the center of the vortex, the phase of the order parameter changes by $2 \pi$, and around antivortex it changes by $-2 \pi$. Thus, we can conclude that these zeros of the order parameter indeed correspond to the vortex and antivortex. Increasing of $\omega$ leads to the motion of vortex toward the system center and antivortex in the opposite direction, where $|\psi|$ is very small. The same is happening along the other two directions. As a result, three vortices penetrate the inner part of a cloud in a symmetrical pattern, whereas three antivortices move far from the cloud center but never disappear. The spot, where vortex-antivortex pair nucleates, is characterized by a small value of $|\psi|$, approximately, fifty times smaller than $|\psi|$ in the cloud center. Therefore, the formation of pairs is invisible on the large length-scale. Also, the interval of $\omega$ between the point of the instability and the vortex state is very narrow, of the order of $10^{-3}$ of trapping frequency. This interval is occupied by the vortex-free state with the order parameter modulated in the azimuthal direction. We believe that vortex-antivortex pairs are not an artifact of our method, since they appear even in the li1 $p<<1$, where Gaussian approximation is very accurate. Also, this mechanism of vortex nucleation is rather natural for the continuos system without any borders, as reflected by the boundary condition (1) for the order parameter. 
The similar scenario for the vortex nucleation is realized at values of $p$ corresponding to $l_{\text {inst }}=5$. In this case, five vortices penetrate the inner part of the cloud and five antivortices move in the opposite direction. Thus, surface modes induce ripples of the order parameter and then ripples are naturally transformed to the same number of vortexantivortex pairs. However, when $l_{\text {inst }}$ is even, $l_{\text {inst }}=4$ or 6 , the scenario is different. At first, $|\psi|$ becomes modulated in the azimuthal direction and with increasing of $\omega$, the minima of $\psi$ become more and more pronounced exactly as at odd $l_{\text {inst }}$. But just before the formation of $l_{\text {inst }}$ vortices this phase suddenly becomes unstable with respect to the nucleation of harmonic with $l=l_{\text {inst }} / 2$. The stationary state after this instability corresponds to the local minimum of energy with $\psi$, which is given by a superposition of harmonics with $l=0, l_{\text {inst }} / 2$, and $l_{\text {inst }}$. At $1 \lesssim p \lesssim 1.5$ $\left(l_{\text {inst }}=4\right)$, this phase is also unstable with respect to the nucleation of the harmonic with $l=1$. This implies that the final state contains one vortex in the center of the cloud and one antivortex at infinity. Thus, according to our model, for even values of $l_{\text {inst }}$, not all ripples of $\psi$ are transformed to vortex-antivortex pairs. Number of these pairs is equal to 3 for $l_{\text {inst }}=6$ and 1 or 2 for $l_{\text {inst }}=4$ depending on $p$. The role of surface modes in this case is to facilitate the penetration of one or more number of vortices to the inner part of the cloud. The similar mechanism for vortex penetration was analyzed in Ref. 7] for the particular case of a quadrupole mode, $l=2$, which facilitates penetration of a single vortex. No antivortices were found in this case, since the Thomas-Fermi boundary was assumed. Our result is more general. It shows that penetration of vortices can be related to the induction of different surface modes depending on $p$. After the cascade of various harmonics generation, multiple-vortex penetration can occur, not necessarily one vortex enters the cloud. In our model, the barrier preventing the separation of vortex and antivortex (or surface barrier in models assuming Thomas-Fermi boundary) is destroyed at critical frequencies slightly exceeding the Landau critical frequency for the surface modes. Thus, our model supports the Anglin's results [11, 12], which were obtained by using completely different method, namely, the hydrodynamic approach taking into account spatial variation of the order parameter in the Thomas-Fermi surface layer.

The mechanism for the vortex nucleation via vortex-antivortex pairs is a specific feature of infinite systems. One can see here some analogy with the BKT transition, where topological defects also nucleate by pairs, and the total topological charge is always zero. In finite systems, topological defects usually penetrate through the surface, but here no surface is assumed, since condition (1) should be fulfilled. Therefore, a natural way to create vortices is via vortex-antivortex pairs, after which antivortices are pushed to the regions with small $|\psi|$, since their presence in regions with high $|\psi|$ is energetically unfavorable. Therefore, antivortices are practically undetectable experimentally. An important consequence of this result is that the topological charge of BEC with boundary condition (1) is always equal to zero. This means that for any stable configuration of vortices in the inner part of the cloud there is a configuration of antivortices at the periphery of the system, where the d2ity of particles is very low. Indeed, using our method we calculated $\psi(r, \varphi)$ for different numbers of vortices and we always found the same number of antivortices in the system. Note that some additional topological defects in regions of small $|\psi|$ were reported in Refs. 14, 15, 16], where int2ive numerical methods were used for the solution of the GP equation. They were called "ghost vortices". The correspondence between the antivortices in our model and "ghost vortices" is an open question.

It can be also predicted that in 3D case vortex loops nucleate in rotating BEC instead of vortex-antivortex pairs. With increasing $\omega$, lateral dim2ions of loops grow up and finally 'vortex' parts of loops penetrate the inner part of cloud and 'antivortex' parts go to the opposite direction. This effect is related to the bending of vortex lines found in $3 \mathrm{D}$ numerical simulations 16, 22. It is also interesting to analyze the stability of knotted GP solutions in this case. In 3D superconductors, vortices also penetrate through the surface not as straight lines, but as half-loops 24, 32]. Physically, this is due to the fact that the vortex energy is proportional to its length and therefore a creation of vortex from the point nucleus is easier than from the line one. BKT transition in 3D case also occurs via the generation of vortex loops 33], see also [34].

Now we briefly discuss the case of finite cloud. We perform the same calculations, but using functions

$$
f_{l}(r)=C_{l}\left[\left(\frac{r}{R_{l}}\right)^{l}+\beta_{l}\left(\frac{r}{R_{l}}\right)^{l+2}\right] \exp \left(-\frac{r^{2}}{2 R_{l}^{2}}-i \phi_{l}\right)
$$

where $\beta_{l}$ is an additional parameter, which is chosen in such a way as to meet the boundary condition $f_{l}\left(R_{b}\right)=0, R_{b}$ is the radius of the system. We found that topological defects nucleate via vortex-antivortex pairs, if $R_{b}$ is approximately two times larger than the Thomas-Fermi radius. With further spinning up of the system, some of antivortices leave the system and the topological charge is not necessarily zero. When $R_{b}$ is less, vortices penetrate via the surface as usual, and no antivortices appear. 


\section{CONCLUSIONS}

We studied a nucleation of topological defects in rotating BEC in 2D case within the Gross-Pitaevskii theory with boundary condition of varnishing of the order parameter in infinity. We found that in this model, with increasing slowly of the rotation frequency, vortex-antivortex pairs appear in the system. Vortices move to the inner part of the cloud and antivortices are pushed to the opposite direction. A number of pairs depends on the gas parameter. Topological charge of the system is always equal to zero, and for any configuration of vortices there is a configuration of antivortices far from the cloud center. For 3D case, we predict that vortex loops nucleate instead of vortex-antivortex pairs. Also studied is the role of surface modes in the process of vortex formation. We revealed two scenarios: either all the ripples of the order parameter induced by surface modes are transformed to the vortices or surface modes facilitate penetration of vortices through the cascade of various harmonics generation. A multiple penetration of vortices is possible. In the finite-size clouds, vortices can either penetrate through the surface or nucleate via vortexantivortex pairs depending on the system size. Vortex nucleation occurs at critical velocities slightly exceeding the Landau critical velocity for the surface modes.

\section{Acknowledgements}

Authors acknowledge useful discussions with Y. Castin, M. Tsubota, T. K. Ghosh, T. Mizushima, and S. Ghosh. W. V. Pogosov is supported by the Japan Society for the Promotion of Science.

\section{References}

[1] J. R. Abo-Shaeer, C. Raman, J. M. Vogels, and W. Ketterle, Science 292, 476 (2001).

[2] K. W. Madison, F. Chevy, W. Wohlleben, and J. Dalibard, Phys. Rev. Lett. 84, 806 (2000).

[3] P. C. Haljan, I. Coddington, P. Engels, and E. A. Cornell, Phys. Rev. Lett. 87, 210403 (2001).

[4] E. Hodby, G. Hechenblaikner, S. A. Hopkins, O. M. Marago, and C. J. Foot, Phys. Rev. Lett. 88, 010405 (2002).

[5] T. Isoshima and K. Machida, Phys. Rev. A60, 3313 (1999).

[6] D. L. Feder, A. A. Svidzinsky, A. L. Fetter, and C. W. Clark, Phys. Rev. Lett. 86, 564 (2001).

[7] M. Kraemer, L. Pitaevskii, S. Stringari, and F. Zambelli, Laser Physics 12, 113 (2002).

[8] S. Sinha and Y. Castin, Phys. Rev. Lett. 87, 190402 (2001).

[9] P. O. Fedichev and G. V. Shlyapnikov, Phys. Rev. A60, R1779 (1999).

[10] A. E. Muryshev and P. O. F edichev, cond-mat/0106462

[11] J. R. Anglin, Phys. Rev. A65, 063611 (2002).

[12] J. R. Anglin, Phys. Rev. Lett. 87, 240401 (2001).

[13] F. Dalfovo and S. Stringari, Phys. Rev. A63, 016601 (2001).

[14] M. Machida, N. Sasa, and H. Matsumoto, J. Low Temp. Phys. 138, 623 (2005).

[15] K. Kasamatsu, M. Tsubota, and M. Ueda, Phys. Rev. A67, 033610 (2003).

[16] K. Kasamatsu, M. 5, N. Sasa, and M. Tsubota, cond-mat/0504603

[17] T. K. Ghosh, Phys. Rev. A69, 043606 (2004).

[18] J. E. Williams, E. Zaremba, B. Jackson, T. Nikuni, and A. Griffin, Phys. Rev. Lett. 88, 070401 (2002).

[19] A. Recati, F. Zambelli, and S. Stringari, Phys. Rev. Lett. 86, 377 (2001).

[20] E. Akkermans and S. Ghosh, J. Phys. B: At. Mol. Opt. Phys 37, S127 (2004).

[21] J. J. Garcia-Ripoll and V. M. Perez-Garcia, Phys. Rev. A63, 041603 (2001).

[22] A. Aftalion and I. Danaila, Phys. Rev. A68, 023603 (2003).

[23] C. P. Bean and J. D. Livingston, Phys. Rev. Lett. 12, 14 (1964).

[24] V. P. Galaiko, Sov. Phys. JETP 23, 878 (1966).

[25] D. S. Rokhsar, Phys. Rev. Lett. 79, 2164 (1997); D. Butts and D. S. Rokhsar, Nature (London) 397, 327 (1999).

[26] G. M. Kavoulakis, B. Mottelson, and C. J. Pethick, Phys. Rev. A62, 063605 (2000).

[27] W. V. Pogosov, R. Kawate, T. Mizushima, and K. Machida, Phys. Rev. A72, 063605 (2005).

[28] V. A. Schweigert, F. M. Peeters, and P. S. Deo, Phys. Rev. Lett. 81, 2783 (1998).

[29] J. J. Palacios, Phys. Rev. B58, R5948 (1998).

[30] S. V. Yampolskii and F. M. Peeters, Phys. Rev. B62, 9663 (2000).

[31] W. V. Pogosov, Phys. Rev. B65, 224511 (2002).

[32] A. V. Samokhvalov, JETP 81, 601 (1995). 
[33] G. A. Williams, Phys. Rev. Lett. 59, 1926 (1987).

[34] F. V. Kusmartsev, Phys. Rev. Lett. 76, 1880 (1996).

\section{FIGURES}

Fig. 1. The dependences of rotation frequences $\omega_{\text {inst }}(l)$, at which the axially-symmetric nonvortex state becomes unstable, on gas parameter $p$ at different values of quantized angular momentum $l$ (Fig. 1 (a)). Fig. 1 (b) shows the resulting function, which is given by minimum of $\omega_{\text {inst }}(l)$ with respect to $l$. Dot lines on Fig. 1 (b) are giudes for eyes indicating the boundaries between regions corresponding to different values of $l_{\text {inst }}$. Dashed line on Fig. 1 (b) is the thermodynamical critical frequency.

Fig. 2. Evolution of the modulus of the order parameter in the vicinity of the spot, where vortex-antivortex pair nucleates. Fig. 2 (a) corresponds to the rotation speed 0.77405, Fig. 2 (b) to 0.77417, and Fig. 2 (c) to 0.77419. Number of particles is $10000, p=1$. 


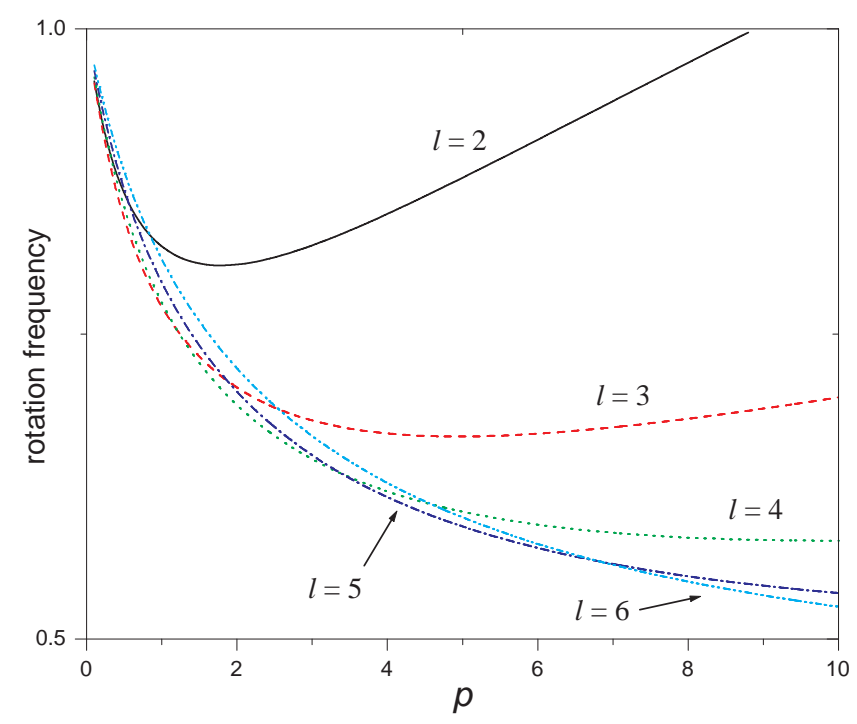




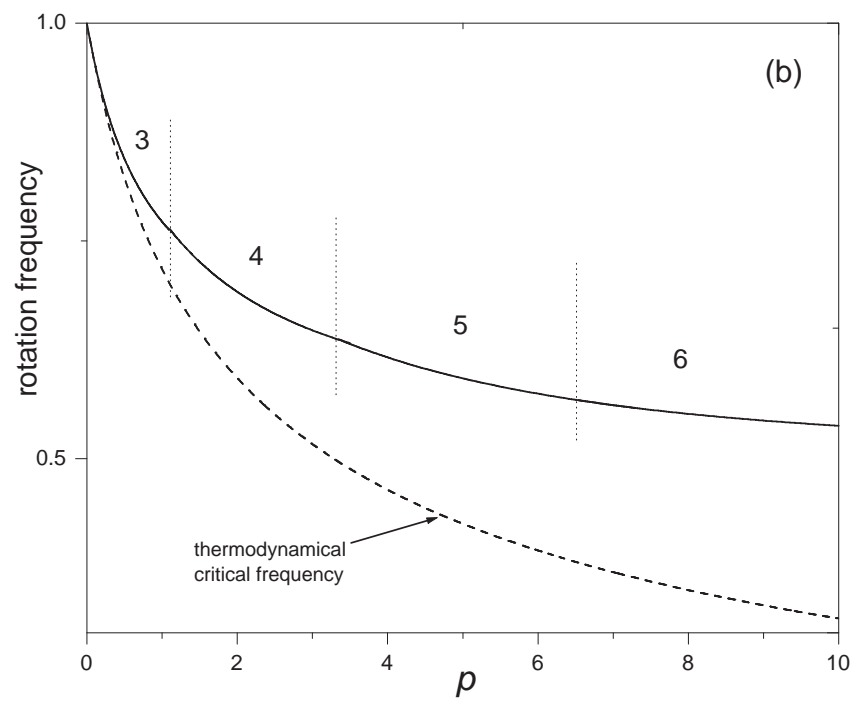




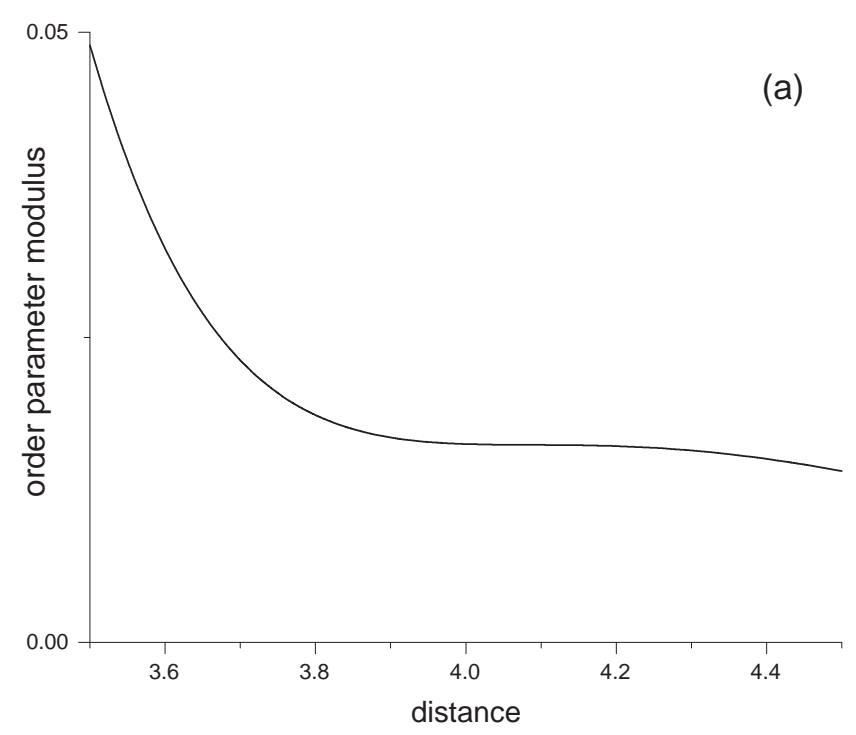




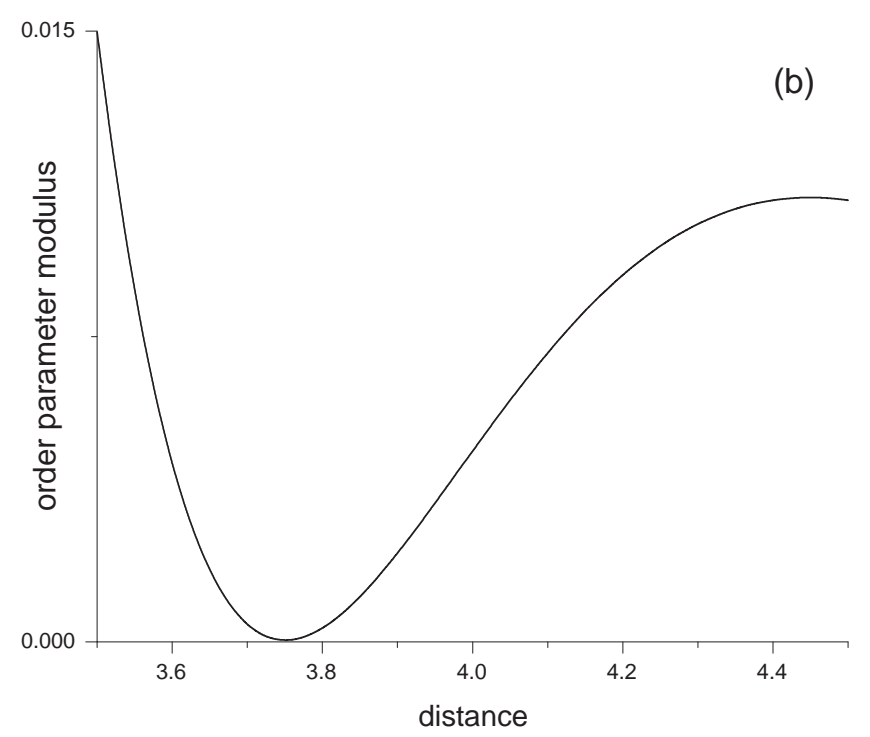




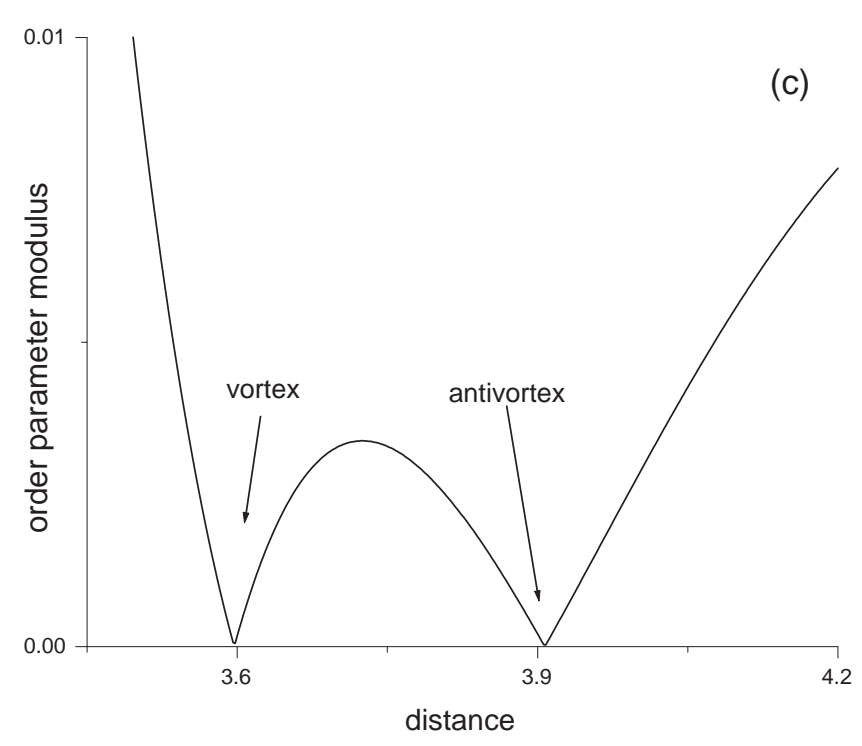

